\title{
Influence of creatinine levels on survival in patients with veno-occlusive disease treated with defibrotide
}

Seom Gim Kong ${ }^{1}$, Je-Hwan Lee ${ }^{2}$, Young Tak Lim³ ${ }^{3}$ Ji Hyun Lee ${ }^{4}$, Hyeon-Seok Eom5 , Hyewon Lee ${ }^{5}$, Do Young Kim ${ }^{6}$, Sung-Nam Lim ${ }^{7}$, Sung-Soo Yoon ${ }^{8}$, Sung-Yong Kim ${ }^{9}$, and Ho Sup Lee ${ }^{10}$

${ }^{1}$ Department of Pediatrics, Kosin University College of Medicine, Busan; ${ }^{2}$ Department of Internal Medicine, Asan Medical Center, University of Ulsan College of Medicine, Seoul; ${ }^{3}$ Department of Pediatrics, Pusan National University School of Medicine, Yangsan; ${ }^{4}$ Department of Internal Medicine, Dong-A Medical Center, Dong-A University College of Medicine, Busan; ${ }^{5}$ Department of Internal Medicine, National Cancer Center, Goyang; ${ }^{6}$ Department of Internal Medicine, Pusan National University Hospital, Busan; ${ }^{7}$ Department of Internal Medicine, Inje University Haeundae Paik Hospital, Busan; ${ }^{8}$ Department of Internal Medicine, Seoul National University Hospital, Seoul; ${ }^{9}$ Department of Internal Medicine, Konkuk University Medical Center, Seoul; ${ }^{10}$ Department of Internal Medicine, Kosin University College of Medicine, Busan, Korea

\section{Influence of creatinine levels on survival in patients with venoocclusive disease treated with defibrotide}

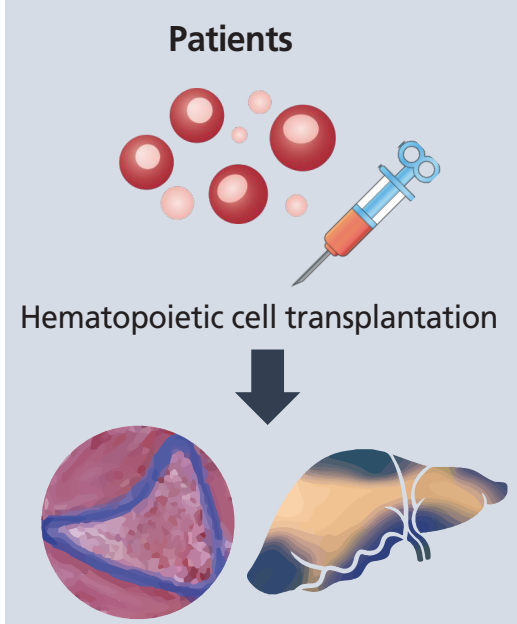

Veno-occlusive disease sinusoidal obstruction syndrome
Outcome

+100 Survival rates

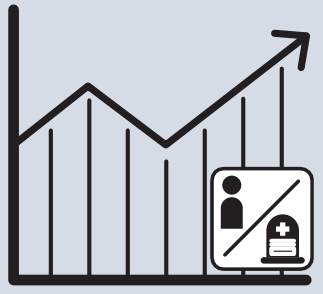

Conclusion

The day +100 survival rate was significantly lower when the Baltimore criteria were satisfied, and when there was an increase in creatinine at the time of defibrotide administration.
Received : April 7, 2021

Revised : May 10, 2021 Accepted: May 10, 2021
Correspondence to Ho Sup Lee, M.D.

Department of Internal Medicine, Kosin University College of Medicine, 262 Gamcheon-ro, Seogu, Busan 49267, Korea

Tel: +82-51-990-5820, Fax: +82-51-990-5820, E-mail: hs52silver@gmail.com https://orcid.org/0000-0001-5974-6884 
Background/Aims: Veno-occlusive disease/sinusoidal obstruction syndrome (VOD/SOS) is one of the most fatal complications of hematopoietic cell transplantation ( $\mathrm{HCT}$ ), and defibrotide is the only curative drug. We conducted this study to confirm the survival rate of VOD/SOS patients diagnosed in Korea and assess the efficacy of defibrotide.

Methods: Patients diagnosed with VOD/SOS after allogenic HCT between 2003 and 2020 were enrolled. We investigated day +100 survival rates and associated risk factors in patients who satisfied the modified Seattle criteria within 50 days of $\mathrm{HCT}$.

Results: A total of 110 patients satisfied the modified Seattle criteria, of which $65.5 \%$ satisfied the Baltimore criteria. Thirty-seven patients were treated with defibrotide. The day +100 survival rate of the 110 patients was $65.3 \%$. The survival rates in patients who did not meet the Baltimore criteria and in those who did were $86.8 \%$ and $53.7 \%$, respectively $(p=0.001)$. The day +100 survival rate of patients treated with defibrotide was $50.5 \%$. Among the patients receiving defibrotide, those whose creatinine levels were more than 1.2 times the baseline had a significantly lower survival rate at $26.7 \%(p=0.014)$. On multivariate regression analysis, the hazard ratio of satisfaction of the Baltimore criteria was 4.54 (95\% confidence interval [Cl], 1.69 to $12.21 ; p=0.003)$. In patients treated with defibrotide, the hazard ratio was $8.70(95 \% \mathrm{Cl}, 2.26$ to $33.45 ; p=$ 0.002 ), when creatinine was more than 1.2 times the baseline on administration.

Conclusions: The day +100 survival rate was significantly lower when the Baltimore criteria were satisfied, and when there was an increase in creatinine at the time of defibrotide administration.

Keywords: Hepatic veno-occlusive disease; Hematopoietic stem cell transplantation; Risk factors; Survival rate; Creatinine

\section{INTRODUCTION}

Hepatic veno-occlusive disease/sinusoidal obstruction syndrome (VOD/SOS) is one of the most fatal complications that can occur in the early stages of hematopoietic cell transplantation (HCT). Activation and damage of sinusoidal endothelial cells are caused by conditioning regimens, such as chemotherapy and radiation therapy, and cytokines produced in damaged tissues [1]. It causes hepatic venous obstruction, portal hypertension, and hepatocellular necrosis, leading to weight gain, water retention, hepatomegaly with tenderness, and jaundice [1]. Its prevalence varies according to the intensity of the conditioning regimen, type of transplantation, and risk factors. A systematic review showed an average prevalence of $13.7 \%$ (0\% to $62.3 \%$ ) [2]. Severe $\mathrm{VOD} / \mathrm{SOS}$ is fatal, with a mortality rate of $80 \%$ or more with supportive treatment alone [2].

Defibrotide was first introduced in the 1950s during research on the development of mammalian organ-derived compounds, and it was first used for VOD/SOS in 1995 [3]. Defibrotide, which has characteristics similar to heparin, binds to vascular endothelial cells and protects the endothelial cells from anticancer drugs, cytokines, and oxidative stress [3]. In addition, it increases the expression of tissue plasminogen activator and thrombomodulin, decreases the expression of von Willebrand factor and plasminogen activity inhibitor-1, and has antithrombotic activity [4]. Many studies have proven its effectiveness on VOD/SOS prevention and treatment [5-9]. In a phase 3 study of defibrotide in patients with severe VOD/SOS, the day +100 survival rate was $38.2 \%$, which was higher than the $25 \%$ of the historical control [6]. The day +100 survival rate of patients with multi-organ dysfunction (MOD) was reported to be $40 \%$ to $50 \%$ according to the International Compassionate-Use Program study and the expanded-access treatment protocol of an investigational new drug (T-IND) study [8,9]. In one study conducted in Korea, 49 HCT patients received defibrotide prophylactically, and only one of them developed VOD/SOS [10].

Recently, it has been reported that earlier administration of defibrotide significantly improved remission and survival rates $[9,11]$. For this reason, rapid diagnosis and early defibrotide treatment have been emphasized [12]. For the rapid diagnosis of VOD/SOS, it is necessary to apply criteria with high sensitivity. Currently, the Baltimore criteria and the modified Seattle criteria are mostly used for VOD/SOS diagnosis, but their sensitivity is not high $[13,14]$. In particular, as the Baltimore criteria includes hyperbilirubinemia, VOD/SOS cannot be diagnosed without jaundice. In addition, for rapid management, it is necessary to complement the guidelines with the timing of defibrotide administration. Since pa- 
tients with mild VOD/SOS are known to improve without treatment, defibrotide has been approved in patients with severe VOD/SOS or MOD. Defibrotide has been approved for severe VOD/SOS in Europe, and for VOD/SOS with renal or pulmonary dysfunction in the United States. However, since VOD/SOS shows a very dynamic course, its mortality remains high even after defibrotide administration, when rapidly progressing to multi-organ failure. In Korea, defibrotide has been approved since 2017, but only receives insurance recognition after it has progressed significantly. We conducted this study to confirm the survival rate of VOD/ SOS patients diagnosed in Korea and the treatment results of defibrotide.

\section{METHODS}

\section{Study populations}

Patients diagnosed with VOD/SOS after allogenic HCT at nine hospitals in Korea between January 2003 and February 2020 were investigated. This study was approved by the Institutional Review Board (IRB) of Kosin University Gospel Hospital (KUGH 2018-06-009). The IRB numbers of the participating hospitals are as follows: 2018-0744 at Asan Medical Center; 05-2021-096 at department of pediatrics of Pusan National University School of Medicine; DAUHIRB-21-108 at Dong-A Medical Center; NCC 2020-0068 at National Cancer Center; 2111-026-109 at Department of Internal Medicine of Pusan National University Hospital; 2021-05-002 at Haeundae Baek Hospital; 2107-116-1235 at Seoul National University Hospital; KUMC 2021-10-056 at Konkuk University Medical Center. Written informed consent by the patients was waived due to a retrospective nature of our study. We investigated age, sex, diagnosis, disease risk at the time of transplantation, performance status, and previous HCT. High disease risk was defined as chronic myelogenous leukemia in the blastic phase, acute leukemia or lymphoma in relapse, and refractory anemia with excess blasts in transformation. Performance status was classified according to the Eastern Cooperative Oncology Group (ECOG) performance status. Conditioning regimen, intensity of conditioning, donor type, and stem cell source were investigated as factors related to $\mathrm{HCT}$.

In addition, we investigated hepatomegaly, right upper quadrant tenderness, ascites, weight gain, and hyperbilirubinemia at the time of diagnosis. This study was conduct- ed in patients who satisfied the modified Seattle criteria within 50 days of transplantation [14]. Of the 124 patients, 110 satisfied the modified Seattle criteria within 50 days of transplantation. We also checked whether the subjects satisfied the Baltimore criteria [13]. If diagnosed more than 21 days after $\mathrm{HCT}$, it was defined as being late-onset. VOD/SOS severity was classified according to the revised criteria from the European Society for Blood and Marrow Transplantation (EBMT) [15]. MOD was defined as renal and/or pulmonary dysfunction.

\section{Treatment}

Total body irradiation (TBI) $\geq 500$ cGy in a single fraction, $\mathrm{TBI} \geq 800$ cGy in a fractionated manner, busulfan $>8 \mathrm{mg} / \mathrm{kg}$ (oral) or $6.4 \mathrm{mg} / \mathrm{kg}$ (intravenously), or melphalan > $150 \mathrm{mg} /$ $\mathrm{m}^{2}$ was defined as myeloablative conditioning (MAC). Most patients who received MAC transplants received $3.2 \mathrm{mg} / \mathrm{kg}$ of busulfan intravenously once daily for 4 days, and $60 \mathrm{mg} /$ $\mathrm{kg}$ of cyclophosphamide intravenously for 2 days. Most patients receiving the reduced-intensity conditioning regimen received $3.2 \mathrm{mg} / \mathrm{kg}$ of busulfan once daily for 2 days and $30 \mathrm{mg} / \mathrm{kg}$ of fludarabine for 6 days. Rabbit anti-thymocyte globulin (ATG; thymoglobulin) was used in half of the patients. ATG was administered at a total dose of 3 to $12 \mathrm{mg} /$ $\mathrm{kg}$, divided equally over 2 to 4 days. For graft-versus-host disease (GVHD) prophylaxis, 33.6\% of patients received cyclosporine alone, $48.2 \%$ received cyclosporine and methotrexate, and $12.7 \%$ received tacrolimus and methotrexate. For VOD/SOS prophylaxis, $37.2 \%$ of patients received heparin alone, $7.3 \%$ received low molecular weight heparin and prostaglandin E1, and 20\% received prostaglandin E1 and ursodeoxycholic acid.

Of the 110 patients, 37 were treated with defibrotide. The median time from the diagnosis of VOD/SOS to the start of defibrotide treatment was 2 days (range, -4 to 25). Seventeen patients were treated within 1 day of diagnosis, and 25 patients were treated after 2 days. The median treatment duration was 12 days (range, 3 to 103), and the dose to be administered was divided into four doses of $25 \mathrm{mg} / \mathrm{kg} / \mathrm{day}$. Seven patients reduced their doses during the treatment.

\section{Outcomes}

We investigated day +100 survival rates and the associated risk factors of patients who satisfied the modified Seattle criteria at diagnosis and of patients treated with defibrotide. For patients treated with defibrotide, the difference in 
survival rates according to the duration from diagnosis to administration, and the laboratory tests at administration, were investigated. Aspartate transaminase (AST), alanine transaminase (ALT), total bilirubin, and creatinine levels were examined before HCT and defibrotide administration. The differences in survival rates were investigated based on: total bilirubin levels of 2 and $5 \mathrm{mg} / \mathrm{dL}$ before the administration of defibrotide; AST and ALT levels that were 2 and 8 times higher than normal levels, respectively; and creatinine levels that were 1.2 and 2 times higher than baseline, respectively.

\section{Statistical methods}

Chi-square and Fisher's exact analyses were performed for categorical variables, and an independent $t$ test was performed for numeric variables. The day +100 survival rate was analyzed using Kaplan-Meier survival analysis, and the difference in survival rate was compared using the log-rank test. The Cox proportional hazards model was used to identify factors affecting the survival rate. All statistical analyses were conducted using SPSS version 25.0 (IBM Co., Armonk, NY, USA). Statistical significance was defined as $p<0.05$.

\section{RESULTS}

\section{Baseline characteristics}

A total of 110 patients satisfied the modified Seattle criteria (Table 1). There were 97 patients (88.2\%) with hyperbilirubinemia, 94 patients $(85.5 \%)$ with hepatomegaly or right upper quadrant tenderness, and 90 patients (81.8\%) with a weight gain of $2 \%$ or more. The median age was 44.0 years (range, 1 to 70 ) and 64 patients (58.2\%) were male. The diagnoses were acute lymphoblastic leukemia in 38 patients (34.5\%), acute myeloid leukemia in 36 patients (32.7\%), and myelodysplastic syndrome in 17 patients (15.5\%). There were 35 patients (31.8\%) with high disease risk, and 20 patients (18.2\%) with an ECOG performance status score of 2 or more. There were 17 patients (15.5\%) who had previously received HCT. Sixty-four patients $(58.2 \%)$ received the MAC regimen. Ninety-three patients (84.5\%) received the HCT with a busulfan-based regimen and six patients (5.5\%) with the TBI-based regimen. Concerning donor-type, a sibling-matched donor was the most common type with 44 patients $(40.0 \%)$, followed by the haploidentical family donor in 30 patients $(27.3 \%)$, an unrelated matched donor in $19(17.3 \%)$, and an unrelated mismatched donor in 17 (15.5\%). As a stem cell source, peripheral blood was used in 73 patients (66.4\%), and bone marrow was used in 37 patients (33.6\%). All patients who used bone marrow had a transplant before 2010.

The median time to VOD/SOS diagnosis was 10.0 days (95\% confidence interval [Cl], 9.7 to 12.5 ) after $\mathrm{HCT}$, and 72 patients $(65.5 \%)$ satisfied the Baltimore criteria at diagnosis. Among the total patients, 82 patients (74.5\%) had painful hepatomegaly, 66 patients $(60.0 \%)$ had ascites, and 58 patients $(52.7 \%$ ) showed a weight gain of more than $5 \%$. According to the revised EBMT criteria for grading of severity, the mild, moderate, severe, and very severe patients were $23(20.9 \%), 60$ (54.5\%), 21 (19.1\%), and five $(5.5 \%)$, respectively. The number of patients with MOD was 49 (44.5\%). A total of 37 patients received defibrotide treatment, and more severe patients were included. At the start of defibrotide treatment, the mean AST, ALT, bilirubin, and creatinine levels were $318 \mathrm{IU} / \mathrm{L}, 215 \mathrm{IU} / \mathrm{L}, 3.5 \mathrm{mg} / \mathrm{dL}$, and $0.97 \mathrm{mg} / \mathrm{dL}$, respectively. There were 24 patients with bilirubin levels above $2 \mathrm{mg} / \mathrm{dL}$, and 15 patients with creatinine levels 1.2 times higher than the baseline.

\section{Survival outcomes}

The day +100 survival rate after HCT in the 110 patients was $65.3 \% \pm 4.6 \%$ (Table 2). The day +100 survival rate of patients under 20 years of age was $90.9 \% \pm 8.7 \%$, and that of adults over 20 years of age was $62.1 \% \pm 4.9 \%(p=0.056)$. The survival rate of patients with high disease-risk was as low as $48.6 \% \pm 8.4 \%(p=0.010)$, and that of patients with an ECOG performance status score of 2 or more was significantly low $(40.0 \% \pm 11.0 \%, p=0.002)$. The survival rates of patients who did not meet the Baltimore criteria and those who were satisfied were $86.8 \% \pm 5.5 \%$ and $53.7 \%$ $\pm 5.9 \%$, respectively $(p=0.001)$ (Fig. 1$)$. According to severity, the survival rates of mild, moderate, severe, and very severe cases were $91.3 \% \pm 5.9 \%, 68.3 \% \pm 6.0 \%, 29.8 \% \pm$ $10.6 \%$, and $50.0 \% \pm 20.4 \%$, respectively ( $p>0.001)$. The survival rate of patients with MOD was as low as $46.1 \% \pm$ $7.2 \%(p<0.001)$.

The day +100 survival rate of patients treated with defibrotide was $50.5 \% \pm 8.3 \%$. The survival rates of patients who were administered defibrotide within 1 and 2 days after diagnosis were $64.7 \% \pm 11.6 \%$ and $37.4 \% \pm 10.1 \%$, respectively $(p=0.106)$. The survival rate was as low as $25.0 \% \pm 15.3 \%$ in patients with total bilirubin levels of 5 
Table 1. Clinical characteristics of VOD/SOS patients

\begin{tabular}{|c|c|c|c|}
\hline Characteristic & No defibrotide $(n=73)$ & Defibrotide $(n=37)$ & $p$ value \\
\hline \multicolumn{4}{|l|}{ Baseline characteristics } \\
\hline \multicolumn{4}{|l|}{ Year } \\
\hline 2003-2010 & $55(75.3)$ & $6(16.2)$ & $<0.001$ \\
\hline 2011-2020 & $18(24.7)$ & $31(83.8)$ & \\
\hline Age, yr & $43.0(38.2-44.6)$ & $43.2(35.6-48.8)$ & 0.281 \\
\hline$<20$ & $5(6.8)$ & $7(18.9)$ & 0.101 \\
\hline$\geq 20$ & $68(93.2)$ & $30(81.1)$ & \\
\hline \multicolumn{4}{|l|}{ Sex } \\
\hline Male & $44(60.3)$ & $20(54.1)$ & 0.532 \\
\hline Female & $29(39.7)$ & $17(45.9)$ & \\
\hline \multicolumn{4}{|l|}{ Diagnosis } \\
\hline ALL & $23(31.5)$ & $15(40.5)$ & 0.349 \\
\hline AML & $25(34.2)$ & $11(29.7)$ & \\
\hline MDS & $14(19.2)$ & $3(8.1)$ & \\
\hline Others & $11(15.1)$ & $8(21.6)$ & \\
\hline High disease risk & $19(26.0)$ & $16(43.2)$ & 0.067 \\
\hline ECOG performance status ( $(\geq 2)$ & $9(12.3)$ & $11(29.7)$ & 0.025 \\
\hline Previous transplantation & $10(13.7)$ & 7 (18.9) & 0.474 \\
\hline \multicolumn{4}{|l|}{ Transplantation } \\
\hline \multicolumn{4}{|l|}{ Intensity of conditioning } \\
\hline RIC or non-myeloablative & $32(43.8)$ & $14(37.8)$ & 0.683 \\
\hline MAC & $41(56.2)$ & $23(62.2)$ & \\
\hline \multicolumn{4}{|l|}{ Conditioning } \\
\hline Busulfan-based & $66(90.4)$ & $27(73.0)$ & 0.002 \\
\hline TBI-based & 0 & $6(16.2)$ & \\
\hline Others & $7(9.6)$ & $4(10.8)$ & \\
\hline \multicolumn{4}{|l|}{ Stem cell source } \\
\hline Peripheral blood & $37(50.7)$ & $36(97.3)$ & $<0.001$ \\
\hline Bone marrow & $36(49.3)$ & $1(2.7)$ & \\
\hline \multicolumn{4}{|l|}{ VOD/SOS } \\
\hline Date of diagnosis, day & $10.0(8.7-11.4)$ & $10.0(9.8-16.4)$ & 0.405 \\
\hline Late onset, $>21$ days & $2(2.7)$ & $8(21.6)$ & 0.002 \\
\hline Baltimore criteria satisfaction & $44(60.3)$ & $28(75.7)$ & 0.108 \\
\hline \multicolumn{4}{|l|}{ VOD/SOS eligibility } \\
\hline Hepatomegaly & $63(86.3)$ & $25(67.6)$ & 0.020 \\
\hline RUQ tenderness & $63(87.5)$ & $25(67.6)$ & 0.012 \\
\hline Ascites & $36(49.3)$ & $30(81.1)$ & 0.001 \\
\hline Weight gain, > 5\% & $33(45.2)$ & $25(67.6)$ & 0.026 \\
\hline Bilirubin, $\geq 2 \mathrm{mg} / \mathrm{dL}$ & $65(89.0)$ & $32(86.5)$ & 0.758 \\
\hline \multicolumn{4}{|l|}{ EBMT criteria of severity } \\
\hline Mild & $18(24.7)$ & $5(13.5)$ & $<0.001$ \\
\hline Moderate & $46(63.0)$ & $14(37.8)$ & \\
\hline Severe & $8(11.0)$ & $13(35.1)$ & \\
\hline Very severe & $1(16.7)$ & $5(13.5)$ & \\
\hline Multi-organ dysfunction & $29(39.7)$ & $20(54.1)$ & 0.153 \\
\hline
\end{tabular}

Values are presented as number (\%) or median (95\% confidence interval).

VOD/SOS, veno-occlusive disease/sinusoidal obstruction syndrome; ALL, acute lymphocytic leukemia; AML, acute myeloid leukemia; MDS, myelodysplastic syndrome; ECOG, Eastern Cooperative Oncology Group; RIC, reduced-intensity conditioning; MAC, myeloablative conditioning; TBI, total body irradiation; RUQ, right upper quadrant; EBMT, European Society for Blood and Marrow Transplantation. 
Table 2. Kaplan-Meier estimates of day +100 survival rate

\begin{tabular}{|c|c|c|c|c|c|c|}
\hline & \multicolumn{3}{|c|}{ Total patients } & \multicolumn{3}{|c|}{ Defibrotide group } \\
\hline & Number & Day +100 survival rate & $p$ value & Number & Day +100 survival rate & $p$ value \\
\hline Overall & 110 & $65.3 \pm 4.6$ & & 37 & $50.5 \pm 8.3$ & \\
\hline \multicolumn{7}{|l|}{ Baseline characteristics } \\
\hline \multicolumn{7}{|l|}{ Age, yr } \\
\hline$<20$ & 12 & $90.9 \pm 8.7$ & 0.056 & 7 & $83.3 \pm 15.2$ & 0.054 \\
\hline$\geq 20$ & 98 & $62.1 \pm 4.9$ & & 30 & $42.5 \pm 9.1$ & \\
\hline \multicolumn{7}{|l|}{ Disease risk at baseline } \\
\hline Low/intermediate & 75 & $73.1 \pm 5.1$ & 0.010 & 21 & $55.9 \pm 11.1$ & 0.455 \\
\hline High & 35 & $48.6 \pm 8.4$ & & 16 & $43.8 \pm 12.4$ & \\
\hline \multicolumn{7}{|l|}{ ECOG performance status } \\
\hline $0-1$ & 90 & $70.9 \pm 4.8$ & 0.002 & 26 & $56.5 \pm 9.9$ & 0.219 \\
\hline$\geq 2$ & 20 & $40.0 \pm 11.0$ & & 11 & $36.4 \pm 14.5$ & \\
\hline \multicolumn{7}{|l|}{ Previous transplantation } \\
\hline No & 93 & $67.5 \pm 4.9$ & 0.168 & 30 & $52.2 \pm 9.3$ & 0.616 \\
\hline Yes & 17 & $52.9 \pm 12.1$ & & 7 & $42.9 \pm 18.7$ & \\
\hline \multicolumn{7}{|l|}{ Transplantation } \\
\hline \multicolumn{7}{|l|}{ Intensity of conditioning } \\
\hline RIC or nonmyeloablative & 46 & $62.9 \pm 7.1$ & 0.399 & 14 & $50.0 \pm 13.4$ & 0.463 \\
\hline MAC & 64 & $67.2 \pm 5.9$ & & 23 & $52.2 \pm 10.4$ & \\
\hline \multicolumn{7}{|l|}{ Conditioning } \\
\hline Busulfan-based & 93 & $68.7 \pm 4.8$ & 0.043 & 27 & $55.1 \pm 9.7$ & 0.046 \\
\hline TBI-based & 6 & $50.0 \pm 20.4$ & & 6 & $50.0 \pm 20.4$ & \\
\hline Others & 11 & $45.5 \pm 15.0$ & & 4 & $25.0 \pm 21.7$ & \\
\hline \multicolumn{7}{|l|}{ VOD/SOS } \\
\hline \multicolumn{7}{|l|}{ Late onset (> 21 days) } \\
\hline No & 100 & $66.8 \pm 4.7$ & 0.419 & 29 & $51.0 \pm 9.4$ & 0.724 \\
\hline Yes & 10 & $50.0 \pm 15.8$ & & 8 & $50.0 \pm 17.7$ & \\
\hline \multicolumn{7}{|l|}{ Baltimore criteria satisfaction } \\
\hline No & 38 & $86.8 \pm 5.5$ & 0.001 & 9 & $77.8 \pm 13.9$ & 0.077 \\
\hline Yes & 72 & $53.7 \pm 5.9$ & & 28 & $41.3 \pm 9.6$ & \\
\hline \multicolumn{7}{|l|}{ EBMT criteria of severity } \\
\hline Mild & 23 & $91.3 \pm 5.9$ & $<0.001$ & 5 & $80.0 \pm 17.9$ & 0.550 \\
\hline Moderate & 60 & $68.3 \pm 6.0$ & & 14 & $50.0 \pm 13.4$ & \\
\hline Severe & 21 & $29.8 \pm 10.6$ & & 13 & $32.3 \pm 14.4$ & \\
\hline Very severe & 6 & $50.0 \pm 20.4$ & & 5 & $60.0 \pm 21.9$ & \\
\hline \multicolumn{7}{|l|}{ Multi-organ dysfunction } \\
\hline No & 61 & $80.3 \pm 5.1$ & $<0.001$ & 17 & $76.5 \pm 10.3$ & 0.007 \\
\hline Yes & 49 & $46.1 \pm 7.2$ & & 25 & $29.1 \pm 9.5$ & \\
\hline \multicolumn{7}{|c|}{ Duration from diagnosis to defibrotide administration, day } \\
\hline$\leq 1$ & & & & 17 & $64.7 \pm 11.6$ & 0.106 \\
\hline$\geq 2$ & & & & 25 & $37.4 \pm 10.1$ & \\
\hline
\end{tabular}

Values are presented as mean \pm standard error.

ECOG, Eastern Cooperative Oncology Group; RIC, red uced-intensity conditioning; MAC, myeloablative conditioning; TBI, total body irradiation; VOD/SOS, veno-occlusive disease/sinusoidal obstruction syndrome; EBMT, European Society for Blood and Marrow Transplantation. 
or higher when defibrotide was administered, but the difference was not statistically significant $(p=0.200)$ (Fig. 2). There was no difference in survival rate according to the AST and ALT levels, when defibrotide was administered. The survival rate of patients whose creatinine levels were more than 1.2 times the baseline value was significantly lower at $26.7 \% \pm 11.4 \%(p=0.006)$.

Thirty-eight patients died within 100 days after HCT. Thirteen (34.2\%) patients died from infection, 11 (28.9\%) from VOD/SOS, six (15.8\%) from disease progression, and three (7.9\%) from acute GVHD. Among the patients who did not use defibrotide, six (30.0\%) patients died due to disease progression. All patients who died from disease progression had acute leukemia, and four patients experienced recurrence before HCT. In the defibrotide group, nine (50.0\%) patients died from infection, and seven (38.9\%) died from VOD/SOS.

\section{Risk factors}

In the univariate Cox regression, the factors affecting the day +100 survival rate among all patients were disease risk, ECOG performance status, conditioning regimen, satisfaction with the Baltimore criteria, severity grade, MOD, and defibrotide treatment (Table 3). On multivariate analysis, age, satisfaction with the Baltimore criteria at diagnosis, and severity grading were significant risk factors. The hazard ratio (HR) of satisfaction of the Baltimore criteria at diagnosis was $4.54(95 \% \mathrm{Cl}, 1.69$ to $12.21 ; p=0.003)$. In patients treated with defibrotide, age, conditioning regimen, donor type, MOD, and creatinine elevation at the start of defibrotide administration were significant risk factors on univariate analysis. On multivariate analysis, the HR was $8.70(95 \% \mathrm{Cl}$, 2.26 to $33.45 ; p=0.002$ ) when creatinine value was more than 1.2 times the baseline, when defibrotide was administered.

\section{DISCUSSION}

In our study, $65 \%$ of VOD/SOS patients satisfied the Baltimore criteria at diagnosis, but their survival rate was significantly lower than those who satisfied only the modified Seattle criteria. On multivariate analysis, HR was 4.54 (95\% $\mathrm{Cl}, 1.69$ to $12.21 ; p=0.003)$. Since hyperbilirubinemia was a prerequisite for the Baltimore criteria, it was mainly diagnosed in advanced conditions when compared to the Seattle criteria. In addition, we found that survival rate was
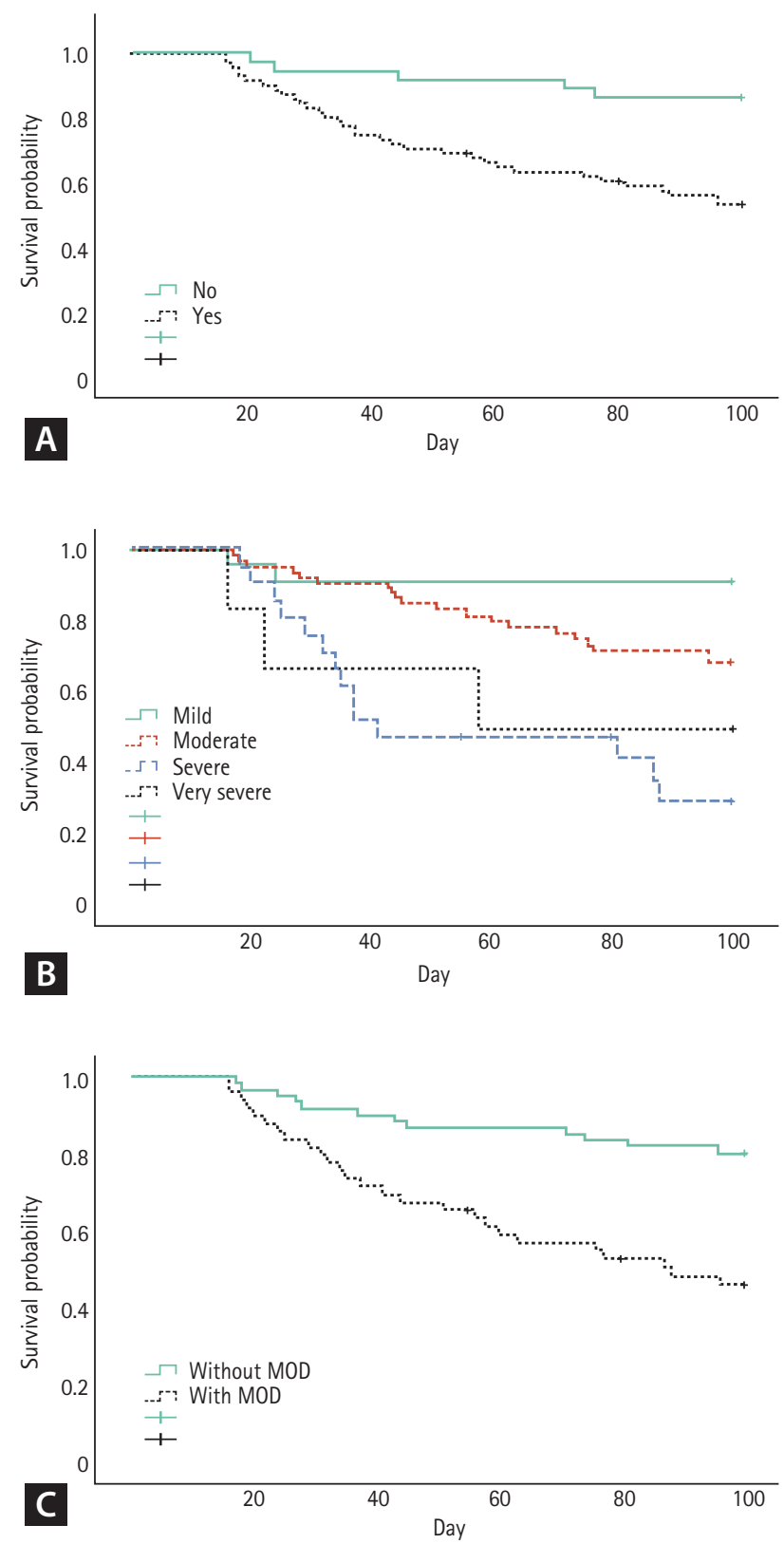

Figure 1. Kaplan-Meier estimated day +100 survival rate $(n=$ 110). (A) Baltimore criteria satisfaction [13]. (B) European Society for Blood and Marrow Transplantation (EBMT) criteria for severity [15]. (C) Multi-organ dysfunction (MOD).

significantly lower when serum creatinine levels at the time of defibrotide administration were 1.2 times higher than the baseline. Serum creatinine levels less than 1.2 times the baseline is considered mild according to the revised EBMT criteria for severity grading [15]. The mortality rate of patients was 8.7 times higher if they had rising creatinine levels before defibrotide administration. 


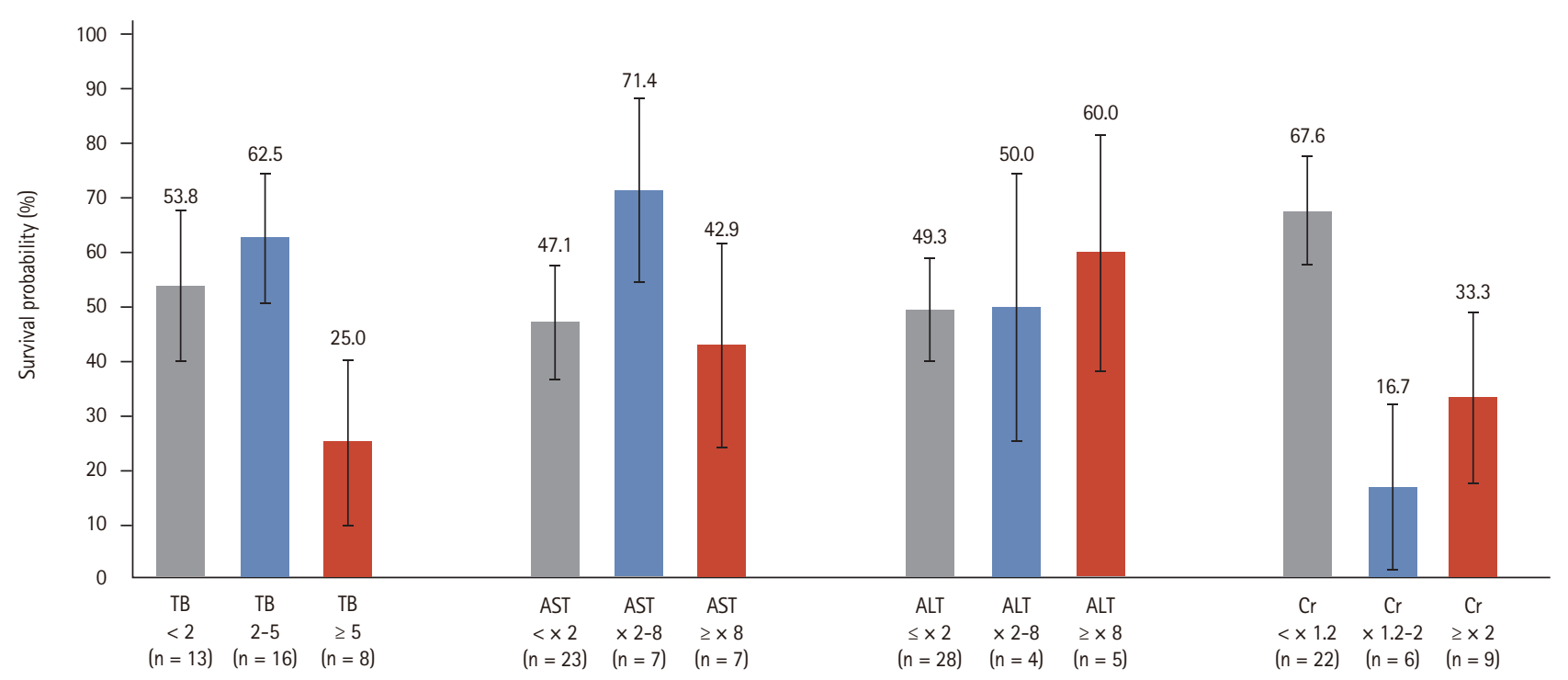

Figure 2. Kaplan-Meier estimated day +100 survival rate according to laboratory findings at the time of defibrotide administration. Total bilirubin ( $T B, p=0.200$ ); aspartate transaminase (AST, $p=0.397)$; alanine transaminase ( $A L T, p=0.822)$; $c r e a t i n i n e ~(C r, p=0.006)$.

The day +100 survival rate for severe VOD/SOS was very poor, approximately $20 \%$ to $30 \%$, with only supportive care prior to defibrotide administration $[2,6,7]$. Defibrotide is currently the only curative drug for severe VOD/SOS. In a recent systematic review on defibrotide, the day +100 pooled survival rate of VOD/SOS was $54 \%$ (35\% to $79 \%$ ) [16]. Furthermore, it was $70 \%$ in patients without MOD and $41 \%$ in patients with MOD. In our study, the overall survival rate of patients with VOD/SOS was $65 \%$, and that of patients who received defibrotide was as low as $50 \%$. We thought this was because severity was higher in patients who received defibrotide. In patients who received defibrotide, the survival rate was $76.5 \%$ in those without MOD and $29.1 \%$ in those with MOD.

In general, it has been known that VOD/SOS without hyperbilirubinemia is rare in adults. However, among patients diagnosed using the modified Seattle criteria in the T-IND study, bilirubin was less than $2 \mathrm{mg} / \mathrm{dL}$ at diagnosis in $29 \%$ of adult patients, and half of these patients were diagnosed within 21 days of transplantation [17]. In patients diagnosed using the modified Seattle criteria in the T-IND study, the day +100 survival rate of patients with bilirubin levels less than $2 \mathrm{mg} / \mathrm{dL}$ at diagnosis was $87 \%$, and that of patients with bilirubin levels of $2 \mathrm{mg} / \mathrm{dL}$ or more was $54 \%$. In other studies comparing the Baltimore criteria and Seattle criteria, meeting the Baltimore criteria was associated with higher severity and lower survival rate $[18,19]$.

Recently, the EBMT published revised criteria for adults and children. The revised EBMT criteria for adults were based on the Baltimore criteria [15]. The revised pediatric EBMT criteria reflected dynamic changes in bilirubin levels, weight gain, and transfusion-refractory thrombocytopenia [20]. Kammersgaard et al. [21] reported that when the revised pediatric EBMT criteria were applied to pediatric VOD/ SOS patients, the diagnosis could be made 3 days earlier. Recently, researchers from MD Anderson Cancer Center retrospectively applied the revised pediatric EBMT criteria to young adults up to the age of 25 [22]. Similarly, in this study, the application of the revised pediatric EBMT criteria was associated with an earlier diagnosis (almost by 2.5 to 3 days). In the past, since there were no effective therapeutic agents for VOD/SOS, strict diagnostic criteria were used. However, since early treatment with defibrotide increases survival rate, it is necessary to apply more sensitive diagnostic criteria. It will be necessary to develop and apply criteria similar to the pediatric EBMT for earlier diagnosis in adults.

Renal dysfunction during VOD/SOS is caused by a hemodynamic situation similar to those of hepatorenal syndrome and renal tubular injury [23]. Fink et al. [23] reported that patients with VOD/SOS without renal dysfunction also had enzymuria, which suggested renal tubular damage. They explained that renal dysfunction and structural damage were 
Table 3. Cox's proportional hazard model for the day +100 survival rate

\begin{tabular}{|c|c|c|c|c|}
\hline & $\begin{array}{l}\text { Univariate HR } \\
\qquad(95 \% \mathrm{Cl})\end{array}$ & $p$ value & $\begin{array}{l}\text { Multivariate HR } \\
(95 \% \mathrm{Cl})\end{array}$ & $p$ value \\
\hline \multicolumn{5}{|l|}{ Total patients $(n=110)$} \\
\hline Recipient age & $1.02(1.00-1.04)$ & 0.054 & $1.04(1.01-1.06)$ & 0.005 \\
\hline Disease risk at baseline (high) & $2.26(1.19-4.27)$ & 0.012 & $1.90(1.88-4.08)$ & 0.101 \\
\hline ECOG performance status ( $\geq 2$ ) & $2.82(1.42-5.60)$ & 0.003 & $1.40(0.62-3.17)$ & 0.419 \\
\hline Previous transplantation & $1.72(0.79-3.75)$ & 0.174 & & \\
\hline Intensity of conditioning (MAC) & $0.76(0.40-1.44)$ & 0.402 & & \\
\hline Late onset (> 21 days) & $1.47(0.57-3.77)$ & 0.422 & & \\
\hline Baltimore criteria satisfaction & $4.35(1.70-11.15)$ & 0.002 & $4.54(1.69-12.21)$ & 0.003 \\
\hline \multicolumn{5}{|l|}{ EBMT criteria } \\
\hline Mild & Reference & & Reference & \\
\hline Moderate & $3.94(0.92-16.92)$ & 0.065 & $4.74(1.03-21.93)$ & 0.046 \\
\hline Severe & $12.02(2.72-53.19)$ & 0.001 & $12.22(2.51-59.49)$ & 0.002 \\
\hline Very severe & $8.34(1.39-49.93)$ & 0.020 & $4.67(0.70-31.14)$ & 0.112 \\
\hline Multi-organ dysfunction & $3.46(1.74-6.88)$ & $<0.001$ & & \\
\hline Defibrotide treatment & $2.27(1.20-4.30)$ & 0.012 & $1.32(0.63-2.77)$ & 0.465 \\
\hline \multicolumn{5}{|l|}{ Defibrotide treatment group $(n=37)$} \\
\hline Recipient age & $1.03(1.00-1.06)$ & 0.030 & $1.05(1.01-1.09)$ & 0.008 \\
\hline Disease risk at baseline (high) & $1.42(0.56-3.58)$ & 0.458 & $2.12(0.47-3.19)$ & 0.690 \\
\hline ECOG performance status ( $\geq 2$ ) & $1.80(0.70-4.65)$ & 0.227 & $1.30(0.46-3.70)$ & 0.628 \\
\hline Previous transplantation & $1.33(0.44-4.04)$ & 0.619 & & \\
\hline Intensity of conditioning (MAC) & $0.70(0.27-1.82)$ & 0.467 & & \\
\hline Baltimore criteria satisfaction & $3.48(0.80-15.18)$ & 0.098 & & \\
\hline \multicolumn{5}{|l|}{ EBMT criteria } \\
\hline Mild & Reference & & & \\
\hline Moderate & $2.79(0.34-22.74)$ & 0.338 & & \\
\hline Severe & $3.82(0.48-30.77)$ & 0.207 & & \\
\hline Very severe & $2.13(0.19-23.49)$ & 0.537 & & \\
\hline Multi-organ dysfunction & $4.36(1.41-13.47)$ & 0.010 & & \\
\hline $\begin{array}{l}\text { Duration from diagnosis to defibrotide } \\
\text { administration ( } \geq 2 \text { days) }\end{array}$ & $2.24(0.80-6.29)$ & 0.127 & $2.07(0.71-6.03)$ & 0.182 \\
\hline \multicolumn{5}{|l|}{ Laboratory finding at administration } \\
\hline Bilirubin, $\geq 5$ mg/dL & $2.38(0.89-6.35)$ & 0.084 & $0.59(0.16-2.18)$ & 0.433 \\
\hline $\mathrm{AST}, \geq 320 \mathrm{IU} / \mathrm{L}$ & $1.28(0.42-3.90)$ & 0.662 & & \\
\hline $\mathrm{ALT}, \geq 320 \mathrm{IU} / \mathrm{L}$ & $0.67(0.15-2.93)$ & 0.598 & & \\
\hline Creatinine, $\geq 1.2 \times$ baseline & $3.10(1.20-8.04)$ & 0.020 & $8.70(2.26-33.45)$ & 0.002 \\
\hline
\end{tabular}

HR, hazard ratio; Cl, confidence interval; ECOG, Eastern Cooperative Oncology Group; MAC, myeloablative conditioning; EBMT, European Society for Blood and Marrow Transplantation; AST, aspartate transaminase; ALT, alanine transaminase. 
common findings of VOD/SOS, even in the absence of elevated creatinine. We were unable to predict whether patients remain in a mild condition or progress; however, Ragoonanan et al. [22] have reported that approximately $80 \%$ of patients had progressed to a severe condition. Therefore, if a significant number of patients eventually progress to a severe condition, defibrotide administration in patients with mild or moderate disease will never be considered excessive. In particular, administration of the drug before progression of renal dysfunction will help improve patient survival.

One of the reasons we are reluctant to actively use defibrotide is due to its side effects. Bleeding, hypotension, and diarrhea are common side effects of defibrotide. However, in a phase III study, the frequency of bleeding and hypotension in the defibrotide group was similar to that in the historical control group [6]. Defibrotide prophylaxis studies showed no significant differences in adverse effects according to administration $[5,24]$. When defibrotide was used prophylactically, there were fewer side effects than when used for treatment. The T-IND study also reported that side effects were lower in patients with bilirubin levels less than 2 $\mathrm{mg} / \mathrm{dL}$ at diagnosis [17]. This suggests that side effects such as bleeding and hypotension may be aggravated by symptoms of advanced VOD/SOS, as apart from being side effects of the drug itself [25]. In addition, several studies have reported that the incidence of grade III/IV acute GVHD was significantly lower in patients receiving defibrotide $[7,26]$.

Our study had limitations as a retrospective study. The reasons for the delay in administration of defibrotide were not analyzed. In general, the reasons for delay in defibrotide administration include uncertainty of diagnosis, delay in diagnosis results, delay in usefulness in medical centers, and active bleeding, in addition to those mentioned above [27]. Therefore, Carreras [12] recommended using defibrotide as soon as possible if VOD/SOS is diagnosed or suspected, and discontinuing it if it is not confirmed later.

In conclusion, the day +100 survival rate was significantly lower when the Baltimore criteria were satisfied on diagnosis of VOD/SOS and when there was an increase in creatinine levels at the time of defibrotide administration. The use of highly sensitive diagnostic criteria and administration of defibrotide before the start of renal dysfunction improved the survival rate.

\section{KEY MESSAGE}

1. The day +100 survival rate of all veno-occlusive disease/sinusoidal obstruction syndrome patients was $65.3 \%$, and that of patients treated with defibrotide was $50.5 \%$

2. In those patients who satisfied the Baltimore criteria at diagnosis, the survival rate was as low as $53.7 \%$ and the hazard ratio (HR) was 4.54 .

3. In patients who already had rising levels of creatinine upon administration of defibrotide, the survival rate was as low as $26.7 \%$, and the HR was 8.70 .

\section{Conflict of interest}

No potential conflict of interest relevant to this article was reported.

\section{Acknowledgments}

The sponsorship for this study was funded by Handok Inc., Seoul, Republic of Korea. The sponsors had no role in study design, data collection and analysis, decision to publish, or preparation of the manuscript.

\section{REFERENCES}

1. Mohty M, Malard F, Abecassis M, et al. Sinusoidal obstruction syndrome/veno-occlusive disease: current situation and perspectives-a position statement from the European Society for Blood and Marrow Transplantation (EBMT). Bone Marrow Transplant 2015;50:781-789.

2. Coppell JA, Richardson PG, Soiffer R, et al. Hepatic veno-occlusive disease following stem cell transplantation: incidence, clinical course, and outcome. Biol Blood Marrow Transplant 2010;16:157-168.

3. Richardson PG, Carreras E, lacobelli M, Nejadnik B. The use of defibrotide in blood and marrow transplantation. Blood Adv 2018;2:1495-1509.

4. Corbacioglu S, Richardson PG. Defibrotide for children and adults with hepatic veno-occlusive disease post hematopoietic cell transplantation. Expert Rev Gastroenterol Hepatol 2017;11:885-898.

5. Corbacioglu S, Cesaro S, Faraci M, et al. Defibrotide for prophylaxis of hepatic veno-occlusive disease in paediatric haemopoietic stem-cell transplantation: an open-label, phase 3 , 
randomised controlled trial. Lancet 2012;379:1301-1309.

6. Richardson PG, Riches ML, Kernan NA, et al. Phase 3 trial of defibrotide for the treatment of severe veno-occlusive disease and multi-organ failure. Blood 2016;127:1656-1665.

7. Strouse C, Richardson P, Prentice G, et al. Defibrotide for treatment of severe veno-occlusive disease in pediatrics and adults: an exploratory analysis using data from the center for international blood and marrow transplant research. Biol Blood Marrow Transplant 2016;22:1306-1312.

8. Corbacioglu S, Carreras E, Mohty M, et al. Defibrotide for the treatment of hepatic veno-occlusive disease: final results from the International Compassionate-Use Program. Biol Blood Marrow Transplant 2016;22:1874-1882.

9. Kernan NA, Grupp S, Smith AR, et al. Final results from a defibrotide treatment-IND study for patients with hepatic veno-occlusive disease/sinusoidal obstruction syndrome. $\mathrm{Br}$ J Haematol 2018;181:816-827.

10. Park M, Park HJ, Eom HS, et al. Safety and effects of prophylactic defibrotide for sinusoidal obstruction syndrome in hematopoietic stem cell transplantation. Ann Transplant 2013; 18:36-42.

11. Corbacioglu S, Greil J, Peters C, et al. Defibrotide in the treatment of children with veno-occlusive disease (VOD): a retrospective multicentre study demonstrates therapeutic efficacy upon early intervention. Bone Marrow Transplant 2004;33:189-195.

12. Carreras E. How I manage sinusoidal obstruction syndrome after haematopoietic cell transplantation. Br J Haematol 2015;168:481-491.

13. Jones RJ, Lee KS, Beschorner WE, et al. Venoocclusive disease of the liver following bone marrow transplantation. Transplantation 1987;44:778-783.

14. McDonald GB, Sharma P, Matthews DE, Shulman HM, Thomas ED. Venocclusive disease of the liver after bone marrow transplantation: diagnosis, incidence, and predisposing factors. Hepatology 1984;4:116-122.

15. Mohty M, Malard F, Abecassis M, et al. Revised diagnosis and severity criteria for sinusoidal obstruction syndrome/veno-occlusive disease in adult patients: a new classification from the European Society for Blood and Marrow Transplantation. Bone Marrow Transplant 2016;51:906-912.

16. Richardson P, Aggarwal S, Topaloglu O, Villa KF, Corbacioglu S. Systematic review of defibrotide studies in the treatment of veno-occlusive disease/sinusoidal obstruction syndrome (VOD/ SOS). Bone Marrow Transplant 2019;54:1951-1962.

17. Corbacioglu S, Kernan NA, Pagliuca A, Ryan RJ, Tappe W, Richardson PG. Incidence of anicteric veno-occlusive dis- ease/sinusoidal obstruction syndrome and outcomes with defibrotide following hematopoietic cell transplantation in adult and pediatric patients. Biol Blood Marrow Transplant 2020;26:1342-1349.

18. Blostein MD, Paltiel OB, Thibault A, Rybka WB. A comparison of clinical criteria for the diagnosis of veno-occlusive disease of the liver after bone marrow transplantation. Bone Marrow Transplant 1992;10:439-443.

19. Carreras E, Diaz-Beya M, Rosinol L, Martinez C, Fernandez-Aviles F, Rovira M. The incidence of veno-occlusive disease following allogeneic hematopoietic stem cell transplantation has diminished and the outcome improved over the last decade. Biol Blood Marrow Transplant 2011;17:1713-1720.

20. Corbacioglu S, Carreras E, Ansari M, et al. Diagnosis and severity criteria for sinusoidal obstruction syndrome/veno-occlusive disease in pediatric patients: a new classification from the European society for blood and marrow transplantation. Bone Marrow Transplant 2018;53:138-145.

21. Kammersgaard MB, Kielsen K, Heilmann C, Ifversen M, Muller K. Assessment of the proposed EBMT pediatric criteria for diagnosis and severity grading of sinusoidal obstruction syndrome. Bone Marrow Transplant 2019;54:1406-1418.

22. Ragoonanan D, Khazal SJ, Wang J, et al. Improved detection of sinusoidal obstructive syndrome using pediatric-AYA diagnostic criteria and severity grading. Bone Marrow Transplant 2021;56:175-184.

23. Fink JC, Cooper MA, Burkhart KM, McDonald GB, Zager RA. Marked enzymuria after bone marrow transplantation: a correlate of veno-occlusive disease-induced "hepatorenal syndrome". J Am Soc Nephrol 1995;6:1655-1660.

24. Chalandon Y, Roosnek E, Mermillod B, et al. Prevention of veno-occlusive disease with defibrotide after allogeneic stem cell transplantation. Biol Blood Marrow Transplant 2004;10:347354.

25. Aziz MT, Kakadiya PP, Kush SM, Weigel K, Lowe DK. Defibrotide: an oligonucleotide for sinusoidal obstruction syndrome. Ann Pharmacother 2018;52:166-174.

26. Tekgunduz E, Kaya AH, Bozdag SC, et al. Does defibrotide prophylaxis decrease the risk of acute graft versus host disease following allogeneic hematopoietic cell transplantation? Transfus Apher Sci 2016;54:30-34.

27. Richardson PG, Smith AR, Triplett BM, et al. Earlier defibrotide initiation post-diagnosis of veno-occlusive disease/sinusoidal obstruction syndrome improves day +100 survival following haematopoietic stem cell transplantation. Br J Haematol 2017;178:112-118. 